REVISIÓN

Rev Colombiana Cienc Anim 2016; 8(Supl):400-410.

\title{
Efecto de la suplementación con zinc y selenio sobre la calidad seminal en cerdos
}

\author{
Effect of supplementation with zinc and selenium on seminal quality in pigs
}

SALAZAR, LUIS ${ }^{1}$ Zoot, CARRILLO-GONZALEZ, DIEGO¹ M.Sc, HERNANDEZ H, DARWIN Ph.D.
${ }^{1}$ Universidad de Sucre - Campus Puerta Verde. Facultad de Ciencias Agropecuarias. Departamento de Zootecnia. Grupo de Investigación en Reproducción y Mejoramiento Genético Animal. Sincelejo. Colombia

\section{Key words:}

Trace minerals; Animal reproduction; Spermatogenesis.

\section{Palabras Clave:}

Microminerales; Reproducción animal; Espermatogénesis.
INFORMACIÓN Recibido: 05-09-2016; Aceptado: 25-11-2016. Correspondencia autor: darwin.hernandez@unisucre.edu.co

\section{Abstract}

The Colombian pig production system has grown considerably in recent years, thanks to the implementation of techniques such as artificial insemination, which allows genetic improvement to increase productivity. However, the management model of semen donors and donors is not adequate, being kept in confinement, fed diets that do not meet the requirements of micronutrients ( $\mathrm{Zn}$ and $\mathrm{Se}$ ) altering seminal quality. Therefore, supplementation models with microminerals, such as Zinc and Selenium, have been proposed. This article compiles, relates and discusses the different effects of supplementation with these microminerals on the different physiological-molecular mechanisms that affect the main seminal characteristics of the pig.

\section{Resumen}

El sistema de producción porcina en Colombia, ha tenido un gran crecimiento en los últimos años, gracias a la implementación de técnicas como la inseminación artificial, la cual permite realizar un mejoramiento genético incrementando la productividad. Sin embargo, el modelo de manejo de los reproductores y donantes de semen no es el adecuado, siendo mantenidos en confinamiento, alimentados con dietas que no suplen los requerimientos de micronutrientes ( $Z n$ y Se) alterando la calidad seminal. Por lo anterior, se han propuesto modelos de suplementación con microminerales, tales como el Zinc y el Selenio. En el presente artículo se recopila, relaciona y discute, los diferentes efectos de la suplementación con estos microminerales, sobre los distintos mecanismos fisiológicomoleculares que afectan las principales características seminales del cerdo. 


\section{Introducción}

La biotecnología de la reproducción incluye un conjunto de técnicas derivadas de la biología celular y molecular destinadas a garantizar la bioseguridad y trazabilidad reproductivas, incrementar el rendimiento reproductivo y asegurar la reproducción asistida (BONET et al., 2006). Por tanto, una de las formas de incrementar la productividad de cualquier empresa porcina consiste en el uso de genética mejorada. El uso de machos reproductores de buen potencial productivo garantizará, en parte, esta eficiencia, ya sean en programas de monta natural o de inseminación artificial (IA). En todo caso, la fertilidad de las piaras depende en gran medida del buen estado del verraco y/o del semen usado en la IA. La IA ofrece ciertas ventajas al compararla con la monta natural: reducción de la transmisión de enfermedades, mejoramiento genético e incremento de la rentabilidad (LÓPEZ, 2012).

La variación en la calidad seminal del verraco está determinada por varios factores como: edad, línea genética, estado nutricional y sanitario, estación o época del año, estimulación sexual y la frecuencia de uso en montas o tiempo entre colectas de semen entre otras. Estos factores influyen de manera directa e indirecta en las principales características seminales, como el volumen del eyaculado, $\mathrm{pH}$ seminal, concentración espermática, movilidad, funcionalidad de la membrana celular, integridad del acrosoma y presencia de espermatozoides anormales (VELÁSQUEZ, 2013). La calidad del semen es extremadamente importante pues una reducción de las características seminales causará deficiencias reproductivas y pérdidas económicas notables teniendo en cuenta que cada cerdo participa en un elevado número de servicios durante el año (PETROCELLI et al., 2015).

Comunmente, el macho reproductor es alimentado con raciones comerciales balanceadas para hembras en gestación o en lactancia, que no cumplen con sus requerimientos nutricionales. Estas restricciones alimenticias que se le imponen al cerdo, pueden llegar a limitar el acceso a micronutrientes (microminerales) requeridos para un óptimo desempeño productivo (AUDET et al., 2004).
Por lo tanto, las deficientes practicas alimenticias y de manejo del verraco, pueden ser causantes de problemas a nivel productivo y reproductivo, además, los sistemas de producción porcina son altamente dependientes de insumos externos y presentan una estructura de costos sensible. Bajo este escenario es importante buscar medidas que minimicen el efecto adverso de cualquier factor que impida alcanzar las metas en términos de parámetros productivos (OBERLENDER et al., 2012), siendo la suplementación con minelares la alternativa que se ha planteado (KOLODZIEJ et al., 2004; WILSON et al., 2004; LÓPEZ et al., 2012; PETROCELLI et al., 2015). Así, el presente artículo se recopila, relaciona y discute, los diferentes efectos de la suplementación con estos microminerales, sobre los distintos mecanismos fisiológico-moleculares que afectan las principales características seminales del cerdo.

Principales minerales que influencian la bioquímica reproductiva del cerdo: Entre los principales oligoelementos se incluyen el hierro, zinc, selenio, molibdeno, manganeso, cromo, cobalto, yodo y cobre. De estos, el zinc y el selenio actúan como componentes o activadores de enzimas que ayudan a la detoxificación de radicales libres, división celular y estabilización de moléculas (YATOO et al., 2013); por lo tanto son considerados los más importantes en la función reproductiva (CHEAH y YANG, 2011; KING'ORI, 2012).

Zinc (Zn): El Zn influye en el crecimiento, desarrollo, reproducción y actividad metabólica, debido a que es el principal constituyente de más de 200 metaloenzimas, envueltas en múltiples funciones orgánicas. Las metaloenzimas contienen cofactores metálicos, que pueden estar directamente enlazados a las proteínas de la enzima o a los grupos no proteicos (grupo prostético) (HOPERT, 2011).

Como constituyente de algunas metaloenzimas, el Zn está envuelto en diversas reacciones enzimáticas asociadas con el metabolismo de carbohidratos, síntesis de proteínas y metabolismo de ácidos nucleicos. Por tanto, es esencial en las células gonadales, donde existe constante crecimiento y división celular. Consecuentemente, algunas funciones reproductivas pueden ser 
afectadas negativamente a causa de deficiencias de Zn (SMITH y AKINBAMIJO, 2000). EI Zn juega un papel importante en la espermatogénesis, en la respuesta a la $\mathrm{LH}$, desarrollo de las células de Leydig y en la producción de esteroides a nivel testicular. Entre los efectos adversos por deficiencias de zinc, caben destacar: atrofia en los túbulos seminíferos y deficiente desarrollo testicular en animales jóvenes, lo que ocasiona falta de libido, transtorna la espermatogénesis y disminuye la fertilidad (KUMAR et al., 2011).

El Zn está relacionado en la defensa del organismo contra las especies reactivas de oxígeno (ROS). Las ROS son moléculas altamente reactivas de radicales libres (especies químicas con un electrón desapareado), generadas como producto del metabolismo celular (FRITZIE et al., 2011). Las células espermáticas exhiben una gran capacidad para generar ROS tales como el peróxido de hidrógeno $\left(\mathrm{H}_{2} \mathrm{O}_{2}\right)$, el anión superóxido $\left(\mathrm{O}_{2}^{-}\right)$y el radical hidroxil $(\mathrm{OH} \bullet)$. Debido a su naturaleza altamente reactiva, las ROS pueden reaccionar con otras moléculas causando oxidación, lo que conlleva a cambios estructurales y funcionales, resultando en daño de la integridad de la célula espermática (BANSAL y BILASPURI, 2010).

Para defenderse del daño producido por las ROS las células somáticas y espermáticas, se valen de múltiples tipos de antioxidantes. Entre estos, se encuentra la superóxido dismutasa (SOD), la cual actúa dismutando el anión $\mathrm{O}_{2}$ - en oxígeno y $\mathrm{H}_{2} \mathrm{O}_{2}$. Para que las funciones catalíticas de la SOD se mantengan, se necesitan moléculas de $\mathrm{Zn}^{++}$(FRITZIE et al., 2011).

El $\mathrm{Zn}$ tiene un efecto marcado sobre el eje hipotálamo-hipófisis-testículo, dónde se ha demostrado que su deficiencia puede alterar la producción de testosterona y consecuentemente la espermatogénesis. En el hipotálamo la hormona liberadora de gonadotropinas $(\mathrm{GnRH})$, juega un papel crítico en la función reproductiva al regular la biosíntesis y secreción de las gonadotropinas. La GnHR necesita del factor de transcripción denominado proteína específica 1 (Specificity Protein 1, Sp1) para la transcripción génica y empezar la síntesis de LH y FSH a nivel de hipófisis. La Sp1 es un factor de transcripción con dominio tipo dedos de Zn. Este dominio se caracteriza por requerir la coordinación de uno o más iones de $\mathrm{Zn}$ para estabilizar la estructura proteíca. Convirtiendose así, en un importante factor de regulación de la expresión génica (ROY et al., 2013). KOCHMAN et al. (1992), demostraron que se pueden aumentar los niveles basales de FSH y LH, al estimular la GnRH hipotalámica, con administración de complejos con iones de $\mathrm{Cu}^{++}$y Zinc ${ }^{++}$. En la hipófisis, el Zn se encuentra localizado en las vesículas secretorias del aparato de Golgi. Por lo que juega un papel importante en la producción y secreción de la LH, FSH y prolactina y estos, a su vez, regulan la producción de testosterona (ROY et al., 2013).

En el testículo, el $Z n$ es un cofactor de la enzima 5 alfa reductasa, necesaria para la conversión de testosterona a en su forma biológica activa denominada alfa dihidrotestosterona (DHT) (ALANI et al., 2015) y también para el matenimiento de los niveles normales de esta hormona (AMIDU et al., 2012).

Selenio (Se): El Selenio inorgánico puede encontrarse en tres estados de oxidación: selenito $\left(\mathrm{Se}^{4+}\right)$, selenato $\left(\mathrm{Se}^{6+}\right)$ y seleniuro $\left(\mathrm{Se}^{2-}\right)$. Estos pueden hallarse, en la ración, en forma inorgánica u orgánica. El selenito de sodio es la forma más comunmente usada en las dietas animales (AHSAN et al., 2014).

El selenio tiene un rol importante en la reproducción del macho, pues es un componente fundamental de la selenocisteína. La selenocisteína es un aminoácido análogo a la cisteína que contiene selenio en vez de azufre y es catalogado, también, como el aminoácido 21 (esencial). La selenocisteína es, a la vez, componente de las selenoproteínas (SALINAS, 2010). Las selenoproteínas más estudiadas e importantes en el sistema reproductor de macho son la GPx1, GPx3, mGPx4, cGPx4 y GPx5, las cuales protegen a las células, contra el daño oxidativo, durante los procesos de mitosis, meiosis y maduración espermática. Otras selenoproteínas, como mGPx4 y snGPx4, sirven como componentes estructurales del espermatozoide maduro (Tabla 1) (AHSAN et al., 2014). 
Tabla 1. Selenoproteinas asociadas con la reproducción del macho (Adaptado de AHSAN et al., 2014).

\begin{tabular}{|c|c|c|c|}
\hline Nomenclatura & Selenoproteína & Localización & Función \\
\hline GPx4 (PHGPx) & Glutatión Peroxidasa & $\begin{array}{l}\text { Membranas Intracelulares, } \\
\text { particularmente los testes. }\end{array}$ & Antioxidante intracelular \\
\hline snGPx4 & GPx4 del núcleo espermático & Núcleo del esperma & $\begin{array}{l}\text { Condensación de la cromatina durante la } \\
\text { espermatogénesis }\end{array}$ \\
\hline mGPx4 & GPx4 mitocondrial & Cápsula mitocondrial y pieza media & $\begin{array}{l}\text { espermatogénesis; componente estructural del } \\
\text { esperma maduro }\end{array}$ \\
\hline cGPx4 & GPx4 citosólico & Testes; epitelio del epidídimo & Antioxidante \\
\hline GPx5 & Encima Gpx4 secretada & Lumen del epidídimo & Actúa reduciendo al peróxido de hidrógeno \\
\hline GPx3 & GPx citosólico & Células del epitelio del epidídimo & Protege al epitelio \\
\hline Gpx1 & GPx citosólico & Epitelio del epidídimo & Propiedades antioxidantes \\
\hline Selenoproteína P & Selenoproteína P del plasma & Plasma sanguíneo & Transporta selenio a las testis. \\
\hline ApoER2 & Apolipoproteína E Receptor-2 & Células de Sertoli, en los testes. & $\begin{array}{l}\text { Receptor del selenio. Hace que el selenio sea } \\
\text { ingresado dentro de las células de Sertoli. }\end{array}$ \\
\hline
\end{tabular}

El selenio se concentra en la cola de los espermatozoides, siendo necesario para mantener de la integridad estructural y función locomotora del mismo (CARRIÓN y MENDEL, 2001); la deficiencia de este mineral puede afectar la estructura y morfología de la cola del esperma y, por tanto, su movilidad y maduración (KUMAR et al., 2011).

Incidencia del Se y Zn sobre las características seminales de cerdos reproductores: Las principales características que se evalúan en los eyaculados son: color, aspecto, $\mathrm{pH}$, el volumen, concentración, movilidad, morfología y vitalidad. Estos parámetros varían de un individuo a otro y son influenciados por muchos factores que se puede clasificar como exógenos (alimentación, régimen de utilización en reproducción, confort, estrés, etc.) o endógenos (genética, endocrinólogos, etc.) (FRUNZĂ et al., 2008). Sin embargo, se considera, que la alimentación es uno de los factores más críticos y la suplementación con zinc y selenio como una alternativa para mejorar dichas características seminales (LECHOWSKY, 2009).

Volumen: Estudios llevados a cabo por diversos autores, con Se como componente mineral primario, muestran mejoras significativas en el volumen de los eyaculados (MARIN-GUZMAN et al., 1997; SOLIS, 2007). En contraste, otros autores exponen que esta no afecta de manera significativa este parámetro (LÓPEZ et al., 2012; THONGCHALAN et al., 2012; LOVERCAMP, 2010). Respecto al Zn, mejoras significativas en este parámetro, son reportados por ESTIENNE y HARPER (2005); HORKÝ et al. (2011); y ARESTOVA y ALEKSEEV (2013).

En general, un aumento en el volumen puede deberse a que el zinc participa en procesos hormonales, estructurales, enzimáticos, y como componente en diversas células y moléculas (OMU et al., 2015). El mineral cumple un papel fundamental en el desarrollo sexual (SHANMUGAM et al., 2015) y la espermatogénesis; las células de Leydig, son responsables de la producción de testosterona. En deficiencia de Zn, las células de Leydig son anormales, con pérdida de tejido epitelial en los túbulos seminíferos de las gónadas (YATOO et al., 2013).

En el eyaculado de cerdo, las secreciones de los testículos y el epidídimo representan 2-4\% del volumen del eyaculado, las de las vesículas seminales, $14-20 \%$, la contribución de las glándulas bulbouretrales es $10-25 \%$, y las de la próstata $55-70 \%$ (BONET et al., 2013).

El zinc también estimula la función prostática, entre el $55-70 \%$ del volumen del eyaculado es aportado por esta glándula (BONET et al., 2013). Esta glándula es la fuente primaria de $\mathrm{Zn}$ en el semen y en ella se encuentran las mayores concentraciones de $\mathrm{Zn}$ del organismo. Otras secreciones prostáticas incluyen grandes cantidades de citrato, fosfatasa ácida, de una enzima parecida a la quimiotripsina y de los cationes de $\mathrm{Ca}^{++}, \mathrm{Mg}^{++}, \mathrm{Zn}^{++}$(KUMAR et al., 2014). El citrato, es un anión trivalente orgánico que tiene diferentes papeles en los tejidos, además 
de ser un agente quelador de los cationes antes mencionados.

Los niveles inusuales de citrato en las céluas de la glándula prostática, son debidos a la baja actividad de la aconitasa mitocondrial (m-aconitasa), la cual previene el metabolismo del citrato a través del ciclo de los ácidos tricarboxílicos. Así, la expresión de la m-aconitasa es regulada por la testosterona y la prolactina, e inhibida por el $\mathrm{Zn}$ (MYCJELKSA et al., 2009). Finalmente los altos niveles acumulados de citrato son secretados como un componente mayor del fluido prostático.

La inhibición de la enzima m-aconitasa con niveles apropiados de $\mathrm{Zn}$, y teniendo en cuenta la influencia que ejerce este elemento en todo el sistema reproductivo del animal, se puede incrementar volumen seminal, a partir del líquido prostático (HEIDEMAN, 2013).

Aspecto: El aspecto o viscosidad del eyaculado varía según el aporte al volúmen final realizado por las glándulas genitales accesorias y de la concentración de espermatozoides (ANDRAVI, 2014). El aspecto del semen está relacionada con la función de la vesícula seminal y la glandula prostática (GONZÁLEZ, 2001).

Las proteínas secretadas por las vesículas seminales representan del 80 al $90 \%$ del total de contenido de las proteínas del plasma seminal, dentro de ellas se encuentras la Semenogelina 1 (SEMG1), la semenogelina 2 (SEMG2) y la fribronectina (BONET et al., 2013). De otro lado, las proteínas de las secreciones prostáticas son de tipo proteasas, principalmente la PSA (Prostatic Specific Antigen).

El $Z n$ induce a las SEMGs a enlazarse unas con otras formando complejos multiméricos insolubles. Este complejo (coágulo), se da por uniones de tipo disulfido los cuales han demostrado tener una gran resistencia. La licuefacción seminal ocurre después de la ruptura de los enlaces disulfido por la enzima PSA. También, se ha sugerido que las SEMGs y sus productos de degradación regula la capacitación espermática (MAL et al., 2015).

Entonces, el Zn es un factor importante para el control de la actividad proteolítica de la PSA, en el plasma seminal. Un posible escenario para la regulación del PSA viene dada de esta forma: el PSA y el Zn son liberados dentro de los conductos prostáticos, con la consecuente inhibición de la enzima por el Zn; luego la mezcla del fluido prostático y el de las vesículas seminales, resulta en la redistribución del $\mathrm{Zn}^{++}$a las SEMG1 y SEMG2 lo cual, al final, resulta en la reactivación del PSA, degradación del coágulo seminal por proteólisis de las SEMGs, y la liberación de las células espermáticas (JONSSON et al, 2005).

pH: Mantener el $\mathrm{pH}$ seminal es un factor muy importante para aumentar la capacidad fertilizadora de las células espermáticas. El pH seminal de los cerdos se encuentra entre $7,3 \mathrm{y}$ 7,9 (FRUNZĂ et al., 2008).

La capacitación espermática, es el primer paso para la activación del esperma hacia la adquisición de la fertilidad, es un mecanismo complejo asociado con una serie de eventos que incluyen incremento intracelular del AMP cíclico (AMPc), calcio, $\mathrm{pH}$ y disminución de la fluidez de membrana (DE LAMIRANDE et al., 2010).

Los fluidos del tracto genital del macho, mantienen el citoplasma espermático en un medio acido $(\mathrm{pH}<6,5)$, el cual, es el mecanismo primario para mantener inmóvil al espermatozoide, antes de la eyaculación. Después de la eyaculación, y durante el tránsito a través del tracto reproductivo de la hembra, la movilidad espermática es iniciada $y$, entonces, hiperactivada para permitir a las células espermáticas penetrar a través del viscoso moco oviductal y las vestimentas protectoras del oocito (LISHKO, et al., 2010). Un pH cercano a la neutralidad, es donde la mayoría de las enzimas en el espermatozoide están activas. Por otro lado, cualquier tipo de desviación hacia la alcalinidad o basicidad, puede reducir la taza metabólica. Un eyaculado acido puede ser asociado con algún tipo de bloqueo de las vesículas seminales y con exceso de moleculas ROS, (OLATUNBOSUN et al., 2013).

Con base en lo anterior, el espermatozoide deberá aumentar la síntesis de SOD, el cual mantiene sus funciones catalíticas en presencia de $\mathrm{Zn}^{++}$ (FRITZIE et al., 2011).

Concentración: Autores que han experimentado con el Se como componente mineral primario en 
una dieta, con resultados positivos en esta variable (MARIN-GUZMAN et al., 1997; KOLODZIEJ y JACYNO, 2004). Investigaciones llevadas a cabo con el $\mathrm{Zn}$, también mostraron aumento en concentración seminal, en la especie porcina (HORKÝ et al., 2011; ARESTOVA y ALEKSEEV, 2013). Sin embargo, los mecanismos exactos por los cuales esto sucede aún no estan claros.

Se da por hecho que la inadecuada ingestión de microminerales, puede tener profundos efectos sobre la fisiología animal, como la poca o nula expresión de algún gen y baja replicación celular (OMU et al., 2015).

El Zn, al ser un componente estructural de los dominios denominados "dedos de zinc", los cuales participan en una variedad de funciones celulares como replicación, reparación, proliferación celular y hacen parte de algunos factores de transcripción (OSREDKAR et al., 2011). La producción espermática necesita una extensa división celular, y es el Zn quién influencia las divisiones mitóticas y meióticas, junto con síntesis de ADN y ARN, al aumentar la actividad de las metaloenzimas involucradas en ese proceso: la polimerasa de ADN y la polimerasa de ARN e inhibidor de la proteína apoptótica Caspase-3 (KUMAR et al., 2006).

El Zn, también hace parte estructural de todos los receptores de andrógenos. Diversos estudios han demostrado que los animales alimentados con dietas deficientes en zinc, presentan baja producción de testosterona y una disminución de los receptores de andrógenos a nivel celular. Esto trae como consecuencia una disminución en la concentración espermática (McDOWELL, 2003).

El Se por su parte, regula la expresión de los genes c-jun y c-fos, constituyentes de la proteína activadora 1 (AP-1). El AP-1 funciona como un transductor o transcriptor de señales para la proliferación celular y es sensible a los ambientes con elevados niveles de oxidación (SHALINI y BANSAL, 2006). Otros genes regulados por la AP-1, son los precursores de antioxidantes como la gamma-glutamil sintetasa ( $\gamma G C S$ ) y la enzima precursora del Glutatión (GSH). El Glutatión (GSH), es un poderoso antioxidante. Esta última es dependiente del Se para poder funcionar y combatir las ROS (AHSAN et al., 2014).
Movilidad: La movilidad espermática evalúa el porcentaje de espermatozoides móviles, así como el tipo de movimiento que presentan. SOLIS, (2007) y TAREQ et al. (2010), demostraron resultados positivos en esta característica en cerdos suplementados con Se y $\mathrm{Zn}$. Sin embargo, los reportes de LÓPEZ et al. (2012), LOVERCAMP, (2013) y THONGCHALAN et al. (2012) no muestran diferencias significativas en la movilidad progresiva e individual.

ElSe se encuentra en forma de selenoporteina GPx4, localizada en la pieza media del espermatozoide, donde cumple un papel antioxidante y estructural (AHSAN et al., 2014). Un aumento en la movilidad, podría explicarse por el efecto del Se como cofactor de la glutatión peroxidasa (GPx). Esta enzima es un poderoso antioxidante que protege a las células espermáticas del daño causado por las ROS (TAREQ et al., 2010). Por otro lado, MARINGUZMAN et al. (2000) demostró que cerdos con dietas bajas en este mineral, producían células espermáticas con baja concentración de ATP, tenían alteraciones en la forma de las mitocondrias de la cola y brechas entre la membrana plasmática y cola, gotas citoplasmáticas, pérdida del contacto entre la membrana mitocondrial con la membrana plasmática, rompimiento de las fibras densas externas (ODF), presencia de una fusión membranal entre la pieza media y la pieza principal y cromatinas parcialmente condensadas, lo que conlleva al rompimiento del ADN. Por otro lado, de dietas con exceso de selenio, se pueden observar piezas medias y colas en un mismo espacio citoplasmático, disminució de la movilidad espermática y efectos adversos en la calidad espermática y fertilidad del macho (SHALINI y BANSAL, 2008).

El Zn interviene en la morfoestructura del espermatozoide. Esta asociado principalmente con las fibras densas externas (ODF) de la cola del espermatozoide. Las ODF son nueve fibras que rodean el axonema por su lado externo, acompañando los microtúbulos en la pieza media y principal, de la cola, por su lado interno. Es comun encontrar al Zn enlazado a grupos sulfhidrilo, de proteínas ricas en cisteína. Los contenidos de $\mathrm{Zn}$ en las ODF son bajos, sin embargo, durante el tránsito del esperma a través del epidídimo, considerables cantidades de $\mathrm{Zn}$ son incorporadas a los espermatozoides, 
provocando una estabilización en las proteínas de estas fibras, debido a la oxidación de los grupos sulfhidrilo, y convirtiendo esos enlaces, en puentes o enlaces disulfuro; la curva flagelar es altamente dependiente del diámetro de las ODF, y su radio es determinado por la dureza de estos elementos estructurales. En la actualidad, la capacidad motriz del espermatozoide no se le asigna a las ODF. Sus funciones principales son, mantener la estructura pasiva elástica y la capacidad de retroceso elástico de la cola, protege a la estructura de la cola contra las fuerzas de choque durante el transporte en el epidídimo durante la eyaculación y brindar soporte al golpe axonémico (fuerza de transmisión) (AHSAN et al., 2014). Las altas concentraciones de $Z n$ existentes en la cola del esperma maduro, permite inferir que este está implicado en la utilización y control de energía por medio del ATP (SHANMUNGAM et al., 2015).

Morfología: La morfología es un parámetro que indica el porcentaje de espermatozoides con defectos estructurales. En la actualidad, quizás lo más adecuado sea identificar cada una de las anormalidades por su ubicación dentro de la estructura del espermatozoide (KUBUS, 2010).

Es sabido que el $\mathrm{Zn}$ y el Se tienen un gran impacto en la integridad morfológica del esperma, especialmente en la formación de la pieza media (CHEAH Y YANG, 2011.). El Zn es transportado hacia las espermatogonias por medio de unos organelos llamados, comúnmente "transportadores de zinc". Durante la espermatogénesis, estos transportadores son los encargados de proveer, en cada nivel espermatogénico, del sustrato necesario para que el proceso se lleve a cabo normalmente. También proveen a las células de Leydig con las cantidades necesarias del mineral, para la producción de testosterona (CROXFORD et al., 2011). Por su parte el selenio es transportado desde el torrente sanguíneo, hasta las células de Sertoli, por la Selenoproteina P, para soportar el proceso de espermatogénesis. Como ya se discutió en apartados anteriores tanto el Zn como el Se tienen un papel importante en la función de los receptores de hormonas y como un modulador de la expresión de ciertos factores de transcripción durante el proceso de la espermatogénesis.
Estos minerales también tienen función antioxidante por acción del GPx contra agentes externos, como las ROS, y hay evidencia que sugiere que la suplementación con selenio, incrementa la actividad de las GPx (MARINGUZMAN et al., 1997).

Vitalidad: Esta característica mide el número o porcentaje de espermatozoides vivos. Estudios hechos por JACYNO y KAWĘCKA. (2002); KOLODZIEJ y JACYNO. (2004) y TAREQ, et al. (2010) sugieren que el Se y el Zn pueden influenciar positivamente el número de células espermáticas viables, en un eyaculado.

El espermatozoide tiene como función transportar un genoma haploide sin daños, hasta el oocito, el espermatozoide ha desarrollado una estructura (cromatina), que es muy diferente a las de las otras células somáticas. La estructura de la cromatina está hecha para que sea extremadamente resistente a condiciones que podrían dañar el ADN. Al mismo tiempo, la cromatina tiene la propiedad de hacer que, rápidamente, el ADN esté disponible en el ooplasma (BJÖRNDAHL y KUVIST, 2010).

El Zn tiene un rol importante en la vitalidad y la prevención de la degradación del espermatozoide y la estabilización de la membrana espermática (AL-ANI et al., 2015). Este es necesario para estabilizar la membrana espermática, al prevenir la oxidación de los grupos sulfhirilo a la forma disulfido. Deficiencias de zinc en el núcleo, podrían desestabilizar la estructura cuaternaria de la cromatina, lo que reduciría la capacidad de fertilización del espermatozoide y llevar a un incremento de defectos de cabeza, los cuales reducen aún más la capacidad fertilizadora del espermatozoide (ZHAO et al., 2016).

Por su parte el Se desempeña el rol de agente antioxidante durante la espermiogénesis. En este paso las histonas somáticas del núcleo espermático son reemplazadas por una serie de proteínas básicas y, al final, por protaminas. Los grupos sulfhidrilo libres en los residuos de las protaminas, son oxidados durante el tránsito de las espermatidas a través del epidídimo. El cambio de histonas somáticas a protaminas básicas (P1 y P2), hace al ADN transcripcionalmente 
inactivo, simultáneamente, con un muy compacto y cristalino empaquetamiento. Esto protege al ADN, reduciendo el acceso de potenciales fuentes de radicales libres (agua), y compuestos solubles en agua que podrían contribuir al daño al ADN; el ADN tiene que estar listo/disponible al llegar al oocito. Un empaquetamiento defectuoso de la cromatina, puede causar compactación reducida o supercompactación. Una compactación disminuida podría incrementar el riesgo de que sustancias externas tengan acceso al ADN, mientras la supercompactación comprometerá el tiempo de entrega del ADN en el ooplasma (BJÖRNDAHL y KUVIST, 2010).

\section{Referencias}

AHSAN, U.; KAMRAN, Z.; SHMAD, S.; BABA, W.; RIZA, W.; IQBAL, Z. 2014. Role Of Selenium In Animal Reproduction - A review. Animal Reproduction Science 146:55-62.

AL-ANI, N.; AL KAWAZ, U.; SAEED, T. 2015. Protective Influence Of Zinc On Reproductive Parameters In Male Rat Treated With Cadmiun. American Journal Of Medicine And Medical Sciences 5 (2):73-81.

AMIDU, N.; OWIREDU, W.; BEKOE, M.; QUAYE, L. 2012. The Impact Of Seminal Zinc And Fructose Concentration On Human Sperm Characteristic. Journal Of Medical And Biomedical Sciences 1 (1):14-20.

ANDRAVI, S. 2014. Applied Andrology In Water Buffalo. CHENOWETH, P.; LORTON, S. (eds), Animal Andrology. Theories And Applications. Ed. CAB International. Londres, Reino Unido.

ARESTOVA, I.; ALEKSEEV, V. 2013. Morphological Analysis Of The Sperms Of breeding Boars Maintained on Nutritional Supplements. Global Veterinaria 11 (1):84-87.

AUDET. I.; LANFOREST. J.P.; MARTINEAU, G.P.; MATTE, J.J. 2004. Effect Of Vitamin Supplements On Soome Aspects Of Performance, Vitamin Status, And Semen Quiality In Boars. Journal OfAnimal Science 82:626-633.

BANSAL, A.; BILASPURI, G. 2010. Impacts of Oxidative Stress and Antioxidants on Semen Functions. Veterinary Medicine International 2011:1-7.

BJÖRNDAHL, L.; KUVIST, U. 2010. Human Sperm Chromatin Stabilization: A Proposed Model Including Zinc Bridges. Molecular Human Reproduction 16 (1):23-29.

BONET, S.; DOLORES, M.; PINART, E.; SANCHOS.; BUSALLEU E.; YESTE M.; CASAS, I. 2006. Biotecnológica De La Reproducción Porcina: Estado actual y futuro de las técnicas de análisis seminal. Anaporc: revista de la Asociación de Porcinocultura Científica 6 (63):18-23.

BONET, S.; GARCÍA, E.; SEPÚLVEDA, L. 2013. The Boar Reproductive System. Págs. 65-107 en: BONET, S.; CASAS, I.; HOLT, W.; YESTE, M., Boar Reproduction. Fundamentals And New Biological Trends. Ed. Springer. Libro en Formato Electrónico.

CARRIÓN, D.; MEDEL, P. 2001. Interacción Nutrición Reproducción En Ganado Porcino. XVII Curso De Especialización FEDNA. España.

CHEAH, Y.; YANG, W. 2011. Functions Of Essential Nutrition For High Quality Spermatogénesis. Advances In Bioscience And Biotechnology 2:182-197.

CROXFORD, T.; MCCORMICK, N.; KELLEHER. 2011. Moderate Zinc Deficiency Reduces Testicular Zip6 And Zip10 Abundance And Impairs Spermatogenesis In Mice. The Journal Of Nutrition 141 (3):359-365. 
DE LAMIRANDE, E.; LAMONTHE, G. 2010. Levels Of Semenogelin In Human Spermatozoa Decrease During Capacitation: Involvement Of Reactive Oxygen Species And Zinc. Human Reproduction 25 (7):1619-1630.

FRITZIE, C.; YAMAGUCHI, S.; MIURA, C.; OHTA, T.; TOZAWA, Y.; IWAI, T.; MIURA, T.2011. Tolerance Of Spermatogonia To Oxidative Stress Is Due To High Levels Of Zinc And Cu/Zn Superoxide Dismutase. PLoS ONE 6 (2):e16938

FRUNZĂ, I.; CERNESCU, H.; KORODOI G. 2008. Physical And Chemical Parameters Of Boar Sperm. Lucrări Stiintifice Medicină Veterinară 41: 631-640.

GONZÁLEZ, F. 2001. Function Of Seminal Vesicles And Their Correlation On Male Fertility. Asian journal of Andrology 3:251-258.

HEIDEMAN, R.2013. The MolecularAnd Cellular Bases OfPhysiological Regulation. KLEIN, B. Cunningham's Textbook Of Veterinary Phisiology. 5 ed. Elsevier-Saunders, St. Louis, Missouri (USA).

HOPPERT, M. 2011. Metalloenzymes. Reitner, J.; Thiel, V, Encyclopedia Of Geobiology. Springer, The Netherlands.

HORKÝ, P.; JANČíKOVÁ, P.; ZEMAN, L. 2011. The Influence Of The Organic And Inorganic Form Of Zinc On Volume Ejaculate, Sperm, Concentration And Percentaje Of Pathologic Sperm. Research In Pigs Breeding (1) 5:22-27.

JACYNO, E.; KAWĘCKA, M. 2002. Influence Of Organic Se + Vitamn E And Organic Se + Vitamin E On Reproductive Performance Of Young Boars. Agricultural And Food Science In Findland 2:175-174.

JONSSON, M.; LINSE, S.; FROHM, B.; LUNDWALL, A.; MALM, J. 2005. Semenogelins I And II Bind Zinc And Regulate The Activity Of Prostate-Specific Antigen. Biochemical Journal 387:447-453.

KING'ORI, A. 2012. The Breeding Boar - Maximizing Productivity. International Journal of livestock Research 2(3):7-14.

KOCHMAN, K., GAJEWSKA, A.; KOLOWSKI, H.; MASIUKIEWICZ.; RSESZOTARSKA, B. 1992. Increased LH And FSH Release From The Anterior pituitary Of Ovaroectomized Rat, In Vivo, By Copper-, Nickel-, And Zinc-LHRH Complexes. Journal Of Inorganic Biochemistry 48:41-46.

KOLODZIEJ, A.; JACYNO, E. 2004. Effect Of Dietary Selenium And Vitamin E Supplementation On Reproductive Performance Of Young Boars. Electronic Journal of Polish Agricultural Universities, Animal Husbandry 7 (1):68-75

KRISHNA, S.; MAJUMDAR, I.; GRISHIN, N. 2003. Structural Classification Of Zinc Fingers. Nucleic Acids Research 31 (2):532-550.

KUBUS, S.A. 2010. Inseminación artificial porcina. Manual práctico para profesionales, Madrid, España

KUMAR, N.; VERMA, R.; SINGH, L.; VARSHNEY, P.; DASS, R. 2006. Effect Of Different Levels And Sources Of Zinc Supplementation On Quantitative And Qualitative Semen Attributes And Serum Testosterone Level In Crossbred Cattle (Bos IndicusxBos Taurus) Bulls. Reproduction Nutrition Development 46: 662-675.

KUMAR, P.; YADAV, B.; YADAV, S. 2014. Effect Of Zinc And Selenium Supplementation On Semen Quality Of Barbari Bucks. Indian Journal Of Animal Research 48 (4):366-369. 
KUMAR, S; KUMAR, A.; ABDUL, W.; KUMAR, D. 2011. Importance Of Micro Minerals On Reproductive Livestock. Veterinary World 4 (5):230-233.

LECHOWSKY, J. 2009. Effect Of Vitamin C On Semen Quality Of Duroc Breed Boars And Their Crossbreds With Hampshire And Pietrain. ANNALES 27(2):12-18.

LISHKO, P.; BOTCHKINA, I.; FEDORENKO, A., KIRICHOK, Y. 2010. Acid Extrusion From Human Spermatozoa Is Mediated by Flagellar Voltage-Gated Proton Channel. Cell 40 (3):327-337.

LÓPEZ, A. 2012. Fresh Boar Semen: Quality Control And Production. Universiteit Gent. Holanda. Disponible en: URL: http://www.rohh.ugent.be/v3/research/phd/2012/LopezRodriguez_A.pdf

LÓPEZ, M.; URBANO, A.; CÁRDENAS, M. 2012. Manual De Laboratorio Para El Análisis De Semen. OmniaScience. DOI: http://dx.doi.org/10.3926/oss.5

LOVERCAMP, K.; STEWART, K.; LIN, X; FLOWER, W. 2013. Effect Of Dietary Selenium On Boar Sperm Quality. Animal Reproduction Science 138:268-275.

MAL, G.; VYAS, S.; SRIVASAN, A.; PATIL, N.; PATHAK, M. 2015. Studies On Liquefaction Time And Proteins Involved In The Improvement Of Seminal Characteristics In Dromedary Camels (Camelus Dromedarius). Scientifica 2016.

MARIN-GUZMAN, J.; MAHAN, D.; CHUNG, Y; PATE, L.; POPE, W. 1997. Effects Of Dietary Selenium And Vitamin E On Boars Performance And Tissue Responses, Semen Quality And Subsequent Fertilization Rates In Mature Gilts. Journal Of Animal Science 75:2994-3003.

MARIN-GUZMAN, J.; MAHAN, D.; WHITMOYER, R. 2000. Effect Of Dietary Selenium And Vitamin E on The Ultraestructure And ATP Concentration Of Boar Spermatozoa, And The Efficacy Of Added Sodium Selenite In Extended Semen On. Journal Of Animal Science 78:1544-1550.

MCDOWELL, R. 2003. Minerals In Animal And Human Nutrition. Second Edition. Ed. Elsevier. Reino De Los Países Bajos.

MYCJELKSA, M.; PATEL, A.; RIZANER, N.; MAZUREK, M.; KEUN, H.; PATEL, A.; GANAPATHY, V.; DJAMGOZ, M. 2009. Citrate Transport And Metabolism In Mamalian Cells. BioEssays 31:10-20.

OBERLENDER, G.; MURGAS, L.; ZANGERONIMO, M.; SILVA, A.; PEREIRA, L.; MUZZI, R. 2012. Comparison Of Two Different Methods For Evaluating Boar Semen Morphology. Archivos Medicina Veterinaria 44:201 -205.

OLATUNBOSUN, S.; ADESEOLU, F. 2013. Seminal Plasma pH, Inorganic Phosphate, Total And lonized Calcium Concentrations In The Assessment Of Human Spermatozoa Function. Journal Clinical And Diagnostic Research 7 (11):2483-2486.

OMU, A.; AL-AZEMI, M.; AL-MAGHREBI, M.; MATHEW, C.; OMU, E.; KEHINDE, E.; ANIM, J.; ORIOWO, M.; MEMON, A. 2015. Molecular Basis For The Effects Of Zinc Deficiency On Spermatogenesis: An experimental Study In The Sprague-Dawley Rat Model. International Journal Of Urology 31 (1):57-64.

OSREDKAR, J.; SUSTAR, N. 2011. Copper and Zinc, Biological Role and Significance Of Copper/Zinc Imbalance. Journal Of Clinical Toxicology. Disponible en URL: http://www.omicsonline.org/copper-and-zincbiological-role-and-significance-of-copper-zincimbalance-2161-0495.S3-001.php?aid=3055 
PETROCELLI, H.; BATISTA, C.; GOSALVEZ J. 2015. Seasonal Variation In Sperm Characteristics In Southern Uruguay. Revista Brasileira de Zootecnia 44 (1):1-7.

PLESSIS, S.; GOKUL, S.; AGARWAL, A. 2013. Semen Hyperviscosity: Causes, Consecuences And Cures. Frontiers In Bioscience 5: 224-231.

ROY, B.; BAGHEL, R.; MOHANTY, T.; MONDAL, G. 2013. Zinc And Male Reproduction In Domestic Animals: A Review. Indian Journal Animal Nutrition 30 (4):339-350.

SALINAS, G. 2010. Bioquímica De La Selenocisteína, el 21er Aminoácido, y Rol De Las Selenoproteínas En La Salud Humana. Mensaje Bioquímico 34:121-133.

SHALINI, S.; BANSAL, M. 2006. Role Of Selenium In Spermatogenesis: Differential Expression Of Cjun And Cfos In Tubular Cells Of Mice Testis 292 (1):27-38.

SHALINI, S.; BANSAL, M. 2008. Dietary Selenium Deficiency As Well As Excess Supplementation Induces Multiple Defects In Epididymal Spermatozoa: Understanding The Role Of Selenium In Male Fertility. International Journal Of Andrology 31:438-449.

SHANMUGAM, M.; PRAKASH, B.; KUMAR, E.; PANDA, K. 2015. Dietary Organic Zinc And Selenium supplementation Improves Semen Quality And Fertility In Layer Breeders. Indian Journal Of Animal Sciences 85 (2):202-204.

SMITH, O.; AKINBAMIJO, O. 2000. Micronutrients And Reproduction In Farm Animals. Animal reproduction Science 60-61:549-560.

SOLIS, K. 2007. Evaluación De La Calidad De Semen De Verracos Utilizados Para Inseminación Artificial Consumiendo Spermax Forte ${ }^{\circledR}$. Tesis de Grado. Universidad Zamorano. Honduras.

TAREQ, K.; SHARMIN, Q.; TAKAGI, Y.; HAMANO, K.; SAWADA, T.; TSUJII, H. 2010. Effect Of Selenium And Vitamin E On Acrosome Reaction In Porcine Spermatozoa. Reproductive Medicine And Biology 9:73-81.

THONGCHALAM, K.; RUKKWAMSUL, T.; CHOMCHAI, S. 2012. Blood And Semen Selenium Concentrations And Semen Quality In Boars Supplemented With Organic Or Inorganic Selenium. Journal Of Animal And Veterinary Advances 11 (5):603-608.

VELÁSQUEZ, C. 2013. Factores Que Influyen En La Calidad y Principales Características Del Verraco. Tesis de Grado. Universidad Nacional José Faustino Sánchez Carrión. Huacho-Perú.

WILSON, M.; ROZEBOOM, K.; CRENSHAW, T. 2004. Boar Nutrition For Optimum Sperm Production. Disponible en: URL: http://citeseerx.ist.psu.edu/viewdoc/download?doi=10.1.1.519.2703\&rep=rep1\&type=pdf

YATOO, M.; SAXENA, A.; DEEPA, P.; HABEAB, B.; DEVI, S.; JATAV, R.; DIMRI, U. 2013. Role Of Trace Elements In Animals: A Review. Veterinarian World 6:963-967.

ZHAO, J.; DONG, Z.; HU, X.; LONG, Z.; LIU, Q.; BISHAO, S.; WANG, Q.; WU, Q.; LI, L. 2016. Zinc Levels In Seminal Plasma And Their Correlation With Male Infertility: A Systematic Review And Meta-analysis. Scientific Reports 2 (6):1-10. 